\title{
Modelling long-memory volatilities with leverage effect: A-LMSV versus FIEGARCH
}

\author{
Esther Ruiz*, Helena Veiga \\ Departamento de Estadística, Universidad Carlos III de Madrid, 28903 Getafe (Madrid), Spain
}

\begin{abstract}
A new stochastic volatility model, called A-LMSV, is proposed to cope simultaneously with leverage effect and long-memory in volatility. Its statistical properties are derived and compared with the properties of the FIEGARCH model. It is shown that the dependence of the autocorrelations of squares on the parameters measuring the asymmetry and the persistence is different in both models. The kurtosis and autocorrelations of squares do not depend on the asymmetry in the A-LMSV model while they increase with the asymmetry in the FIEGARCH model. Furthermore, the autocorrelations of squares increase with the persistence in the A-LMSV model and decrease in the FIEGARCH model. On the other hand, if the correlation between returns and future volatilities is negative, the autocorrelations of absolute returns increase with the magnitude of the asymmetry in the FIEGARCH model while they decrease in the A-LMSV model. Finally, the cross-correlations between squares and original observations are, in general, larger in absolute value in the FIEGARCH model than in the A-LMSV model. The results are illustrated by fitting both models to represent the dynamic evolution of volatilities of daily returns of the S\&P500 and DAX indexes.
\end{abstract}

.Keywords: Autocorrelations of squares and of absolute values; Conditional heteroscedasticity; Kurtosis; EMM estimator

\section{Introduction}

One of the main empirical characteristics of financial returns is the dynamic evolution of their volatilities. There are two important properties that characterized this evolution. First of all, power transformations of absolute returns have significant autocorrelations which decay toward zero slower than in a short-memory process. Many authors have argued that this pattern of the sample autocorrelations suggests that the volatilities of financial returns can be represented by long-memory processes; see Ding et al. (1993) and Lobato and Savin (1998) among many others. The second property that characterizes volatilities is their asymmetric response to positive and negative returns. This property, known as leverage effect, was first described by Black (1976).

There are two main families of econometric models proposed to represent the dynamic evolution of volatilities. The ARCH-type models are mainly characterized by specifying the volatility as a function of powers of past absolute returns and, consequently, the volatility can be observed one-step ahead. On the other hand, stochastic volatility (SV) models specify the volatility as a latent variable that is not directly observable. There have been several proposals of ARCH-type models that represent simultaneously leverage effect and long-memory. For example, Hwang (2001) generalizes the 
long-memory FIGARCH model of Baillie et al. (1996) to represent the leverage effect. However, Davidson (2004) shows that the FIGARCH model has the unpleasant property that the persistence of shocks to volatility decreases as the long-memory parameter increases. A similar conclusion appears in Zaffaroni (2004) who shows that the FIGARCH model cannot generate autocorrelations of squares with long-memory. Finally, Ruiz and Pérez (2003) showed that the model proposed by Hwang (2001) has identification problems. Consequently, in this paper, we focus on the fractionally integrated EGARCH (FIEGARCH) model proposed by Bollerslev and Mikkelsen (1996) which extends the asymmetric EGARCH model of Nelson (1991) to long-memory. Following the arguments of He et al. (2002) for the short-memory EGARCH model, we derive the kurtosis, autocorrelations of squares and absolute observations and the cross-correlations between returns and powers of absolute returns of the $\operatorname{FIEGARCH}(1, d, 0)$ model with Gaussian errors.

Alternatively, in the context of SV models, Harvey (1998) and Breidt et al. (1998) have independently proposed long-memory stochastic volatility (LMSV) models in which the underlying log-volatility is modelled as an ARFIMA process. On the other hand, Harvey and Shephard (1996) propose to model the leverage effect of the short-memory SV model by introducing correlation between the noises of the level and volatility equations. Recently, Yu et al. (2006) have also proposed an extension of this asymmetric SV model in which a Box-Cox transformation of the volatility follows an AR(1) process. This model encompasses several popular SV models as special cases. However, the statistical properties of the new model are still unknown. So et al. (2002) have proposed a threshold SV model which is also able to represent the leverage effect. In this paper, we focus on the proposal of Harvey and Shephard (1996) and propose the Asymmetric LMSV (A-LMSV) model that generalizes the LMSV model to cope with leverage effect. Assuming that the log-volatility is an ARFIMA $(1, d, 0)$ model and Gaussianity of the errors, we derive the statistical properties of the new model and compare them with the properties of the FIEGARCH model. We show that both models explain in different ways the kurtosis and correlations of absolute and squared returns. However, the cross-correlations between returns and powers of absolute returns behave in a similar fashion.

The rest of the paper is organized as follows. The description of the statistical properties of the A-LMSV $(1, d, 0)$ model is done in Section 2. In Section 3, we derive the properties of the $\operatorname{FIEGARCH}(1, d, 0)$ model and compare them with the properties of the A-LMSV model. Section 4 contains an empirical illustration by fitting both models to daily financial returns of the S\&P500 and the DAX indexes. Section 5 concludes the paper.

\section{Asymmetric LMSV models}

The LMSV model, proposed independently by Harvey (1998) and Breidt et al. (1998), extends the stochastic volatility model of Taylor (1982) by assuming that the volatility follows a weakly stationary fractional integrated process. Therefore, the LMSV model captures the long-memory property often observed in the powers of absolute returns. In this section, we extend the LMSV model to represent the asymmetric response of volatility to positive and negative returns. Following Taylor (1994) and Harvey and Shephard (1996), this asymmetry is introduced by allowing the disturbances of the level and volatility equations to be correlated. In this paper, we consider that the log-volatility is specified as an ARFIMA $(1, d, 0)$ process. In this case, the A-LMSV model is given by

$$
\begin{aligned}
& y_{t}=\sigma_{*} \sigma_{t} \varepsilon_{t}, \\
& (1-\phi L)(1-L)^{d} \log \sigma_{t}^{2}=\eta_{t},
\end{aligned}
$$

where $y_{t}$ is the return at time $t$ and $\sigma_{t}$ is its volatility. The parameter $\sigma_{*}$ is a scale parameter and $L$ is the lag operator such that $L x_{t}=x_{t-1}$. The disturbances $\left(\varepsilon_{t}, \eta_{t+1}\right)^{\prime}$ are assumed to have the following bivariate normal distribution:

$$
\left(\begin{array}{c}
\varepsilon_{t} \\
\eta_{t+1}
\end{array}\right) \sim \operatorname{NID}\left(\left(\begin{array}{l}
0 \\
0
\end{array}\right),\left(\begin{array}{cc}
1 & \delta \sigma_{\eta} \\
\delta \sigma_{\eta} & \sigma_{\eta}^{2}
\end{array}\right)\right),
$$

where $\delta$, the correlation between $\varepsilon_{t}$ and $\eta_{t+1}$, induces correlation between the returns, $y_{t}$, and the variations of the volatility one period ahead, $\sigma_{t+1}-\sigma_{t}$. The dynamic properties of the symmetric LMSV model are described by Ghysels et al. (1996). In particular, the stationarity of $y_{t}$ depends on the stationarity of the log-volatility, $h_{t}=\log \sigma_{t}^{2}$. Therefore, if $|\phi|<1$ and $d<0.5, y_{t}$ is stationary. In this case, they show that the variance and kurtosis of $y_{t}$ are given by

$$
\operatorname{Var}\left(y_{t}\right)=\sigma_{*}^{2} \exp \left\{\frac{\sigma_{h}^{2}}{2}\right\}
$$


and

$$
\kappa_{y}=\frac{E\left(y_{t}^{4}\right)}{E\left(y_{t}^{2}\right)^{2}}=3 \exp \left(\sigma_{h}^{2}\right)
$$

respectively, where $\sigma_{h}^{2}=\sigma_{\eta}^{2} \frac{\Gamma(1-2 d)}{[\Gamma(1-d)]^{2}} \frac{F(1,1+d ; 1-d ; \phi)}{(1+\phi)}, \Gamma(\cdot)$ is the gamma function and $F(\cdot, \cdot ; \cdot ; \cdot)$ is the hypergeometric function; see Hosking (1981) for the expressions of the variance and autocorrelation function (acf) of an ARFIMA process. From (4), it can be shown that, given $\sigma_{\eta}^{2}$ and $\phi$, the kurtosis of returns, $\kappa_{y}$, increases with $d$, the parameter of fractional integration. On the other hand, introducing correlation between $\varepsilon_{t}$ and $\eta_{t+1}$ does not change the marginal moments of $y_{t}$ with respect to the symmetric model with $\delta=0$; see Harvey and Shephard (1996) for the model with $d=0$. Therefore, expressions (3) and (4) are also the variance and kurtosis of $y_{t}$ in the A-LMSV model.

Furthermore, although the series of returns, $y_{t}$, is a martingale difference and, consequently a serially uncorrelated sequence, it is not an independent sequence. Note that if the asymmetry is introduced as in Jacquier et al. (2004) by a correlation between $\varepsilon_{t}$ and $\eta_{t}$, the series $y_{t}$ is not a martingale difference; see Harvey and Shephard (1996) and $\mathrm{Yu}$ (2004). There are nonlinear transformations of returns, as for example, powers of absolute returns, which are correlated. After some very tedious algebra, and assuming that $\varepsilon_{t}$ and $\eta_{t}$ are Gaussian and mutually independent, we derive the following expression of the acf of $\left|y_{t}\right|^{c}$ for $c=1$ and 2:

$$
\rho_{c}(k)=\frac{\exp \left(\frac{c^{2}}{4} \sigma_{h}^{2} \rho_{h}(k)\right)\left(1+\left(\delta \sigma_{\eta}\right)^{c}\left(\frac{4}{\kappa_{c}}\right)^{(c-2) / 2} \lambda_{k}^{c}\right)-1}{\kappa_{c} \exp \left(\frac{c^{2}}{4} \sigma_{h}^{2}\right)-1}, \quad k \geqslant 1,
$$

where $\rho_{h}(k)$ is the acf of the log-volatility given by

$$
\rho_{h}(k)=\left(\prod_{i=0}^{k-1} \frac{d+i}{1-d+i}\right) \frac{F(1, d+k ; 1-d+k ; \phi)+F(1, d-k ; 1-d-k ; \phi)-1}{(1-\phi) F(1,1+d ; 1-d ; \phi)},
$$

$\kappa_{c}=\frac{E\left(\left|\varepsilon_{\varepsilon}\right|^{2 c}\right)}{\left\{E\left|\varepsilon_{t}\right|^{c}\right\}^{2}}=\frac{\Gamma(c+0.5) \Gamma(0.5)}{\left[\Gamma(0.5(c+1)]^{2}\right.}$ which takes values $\kappa_{1}=\pi / 2$ and $\kappa_{2}=3$, respectively. Finally, if $d>0, \lambda_{k}=$ $\sum_{i=0}^{k-1} \frac{\Gamma(i+d)}{\Gamma(i+1) \Gamma(d)} \phi^{k-i-1}$. Note that when $\delta=0$, expression (5) becomes the acf of $\left|y_{t}\right|^{c}$ derived by Harvey (1998) for symmetric LMSV models. The expression of the autocorrelations of $\left|y_{t}\right|^{c}$ in (5) is also valid for non-Normal distributions of the errors $\varepsilon_{t}$ as far as $\delta=0$; see Ghysels et al. (1996). The only difference is that the value of $\kappa_{c}$ depends on this distribution. On the other hand, when $d=0, \lambda_{k}=\phi^{k-1}$ and expression (5) becomes the acf of a short-memory SV model with leverage effect. Taylor (1994) have obtained this expression for $c=2$.

The acf in expression (5) depends on the parameters $d, \phi$ and $\sigma_{\eta}^{2}$ that affect both the variance and the acf of the underlying log-volatility process, $\sigma_{h}^{2}$ and $\rho_{h}(k)$, respectively. The autocorrelations also depend on the correlation between $\varepsilon_{t}$ and $\eta_{t+1}, \delta$, and on the power parameter, $c$. For fixed values of the parameters $d, \sigma_{\eta}^{2}$ and $\phi$, the effect of the asymmetry, $\delta$, on the autocorrelation of order $k$ of $y_{t}^{2}$ is measured by $\left(\delta \sigma_{\eta} \lambda_{k}\right)^{2}$. First of all note that this effect is the same regardless of the sign of $\delta$. Furthermore, in empirical applications, the variance of the log-volatility process and the constant $\lambda_{k}$ are typically very small and consequently, this effect is also rather small. Therefore, the autocorrelations of squared returns generated by symmetric and asymmetric LMSV processes are very similar; see Carnero et al. (2004) for a similar result in the context of short-memory ARSV models. As an illustration, the right column of Fig. 1 plots the acfs of squared returns, for the following four A-LMSV models: $\left\{\phi=0, d=0.49, \sigma_{\eta}^{2}=0.05\right\},\{\phi=0, d=0.49$, $\left.\sigma_{\eta}^{2}=0.1\right\},\left\{\phi=0.5, d=0.49, \sigma_{\eta}^{2}=0.1\right\}$ and $\left\{\phi=0.98, d=0, \sigma_{\eta}^{2}=0.05\right\}$. For each of these models, we consider $\delta=0,-0.2,-0.5$ and -0.8 . These models have been chosen to resemble the parameter values often estimated when the LMSV model is fitted to time series of financial returns; see, for example, Pérez and Ruiz (2001). Fig. 1 shows that, for the four models considered, the acfs of squares are nearly the same regardless of the value of the asymmetry parameter $\delta$. 
(phi=0, $d=0.49$, vareta $=0.05$ )

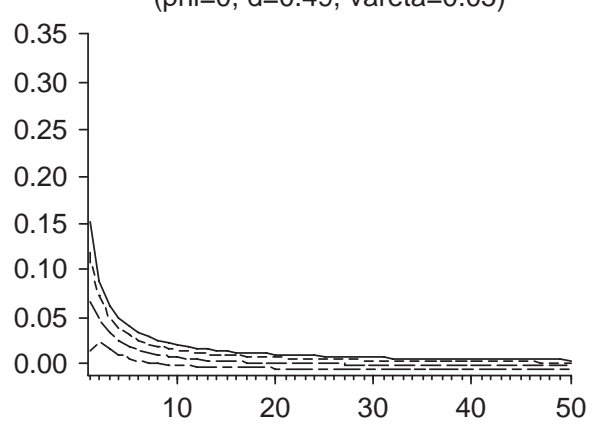

(phi $=0, d=0.49$, vareta $=0.1$ )

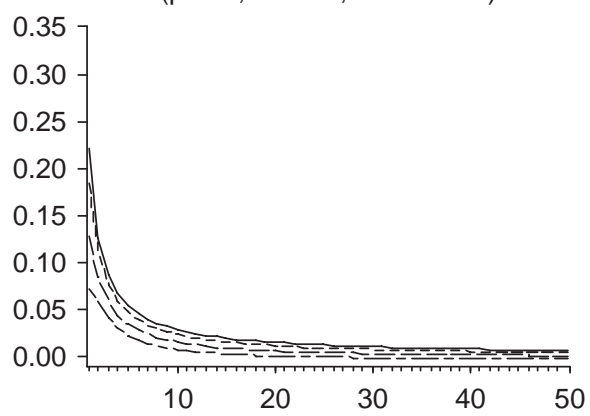

(phi $=0.5, d=0.49$, vareta $=0.1$ )

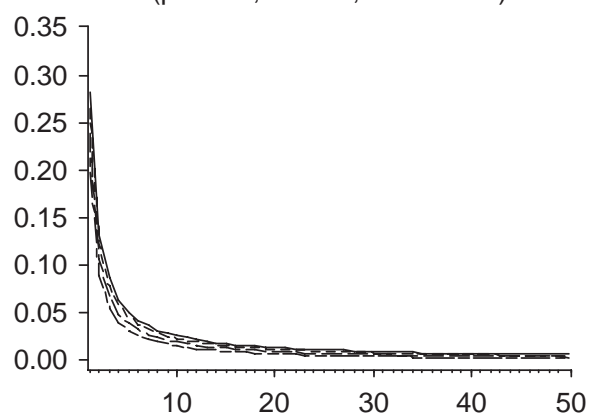

(phi $=0.98, d=0$, vareta $=0.05$ )

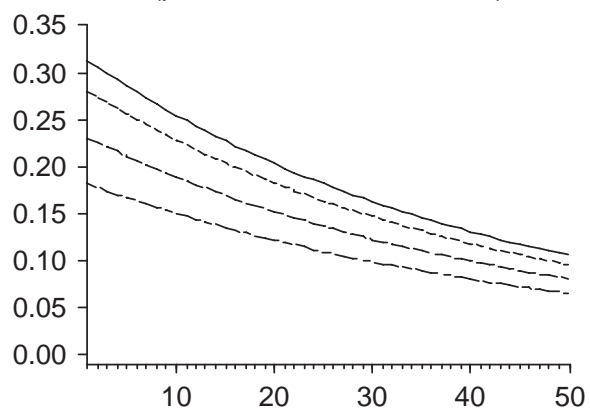

(phi=0, $d=0.49$, vareta $=0.05$ )

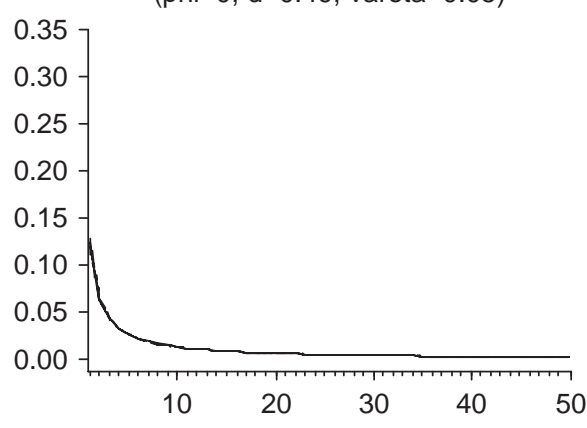

(phi $=0, d=0.49$, vareta $=0.1$ )

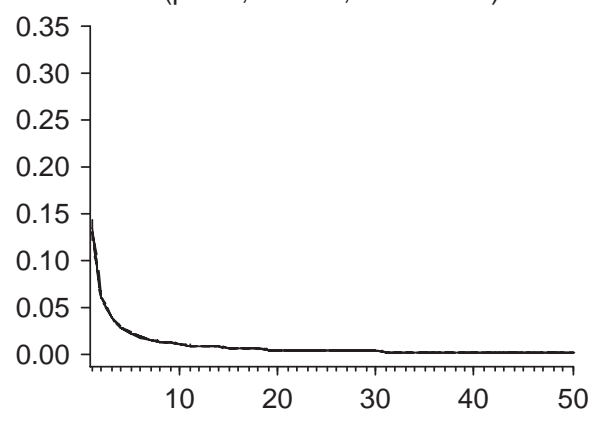

(phi $=0.5, d=0.49$, vareta $=0.1$ )

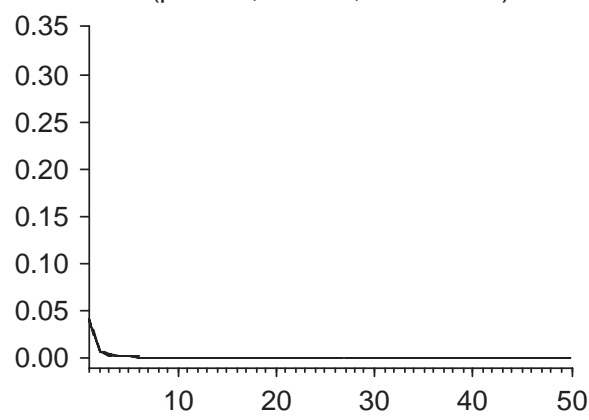

(phi $=0.98, d=0$, vareta $=0.05$ )

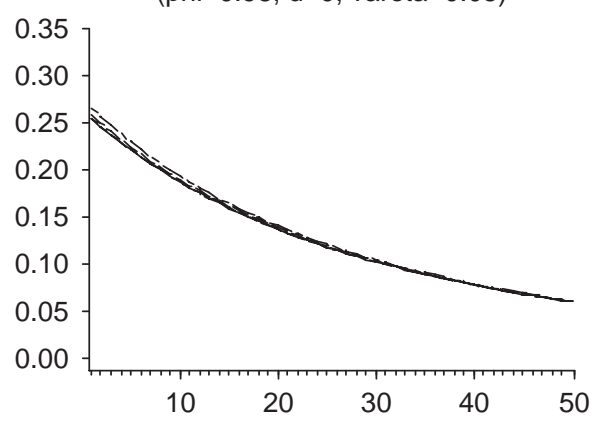

Fig. 1. Autocorrelations of $\left|y_{t}\right|$ (left column) and $y_{t}^{2}$ (right column) in four A-LMSV models with different values of the asymmetric parameter: continuous $(\delta=0)$, dotted $(\delta=-0.2)$, dashed $(\delta=-0.5)$ and dotted-dashed $(\delta=-0.8)$.

We consider now the effect of $\delta$ on the autocorrelation of order $k$ of absolute observations which is measured by $0.627 \delta \sigma_{\eta} \lambda_{k}$. Note that, in this case, a negative correlation between $\varepsilon_{t}$ and $\eta_{t+1}$ decreases the autocorrelations. Depending on the parameters that govern the dynamic evolution of the volatility, the autocorrelations of absolute returns can even be 
(phi $=0, d=0.49$, vareta $=0.05)$

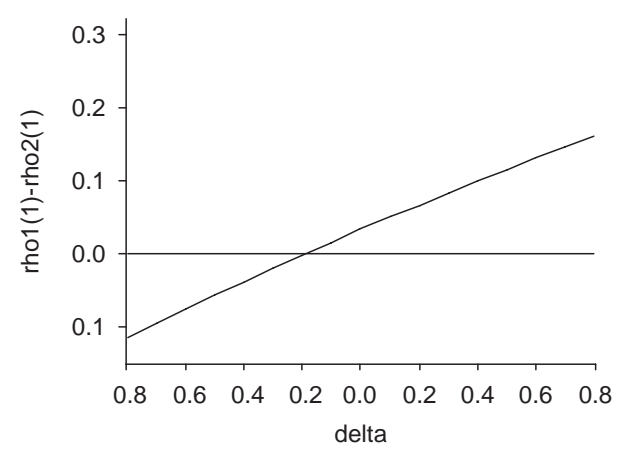

$(p h i=0.5, d=0.49$, vareta $=0.1)$

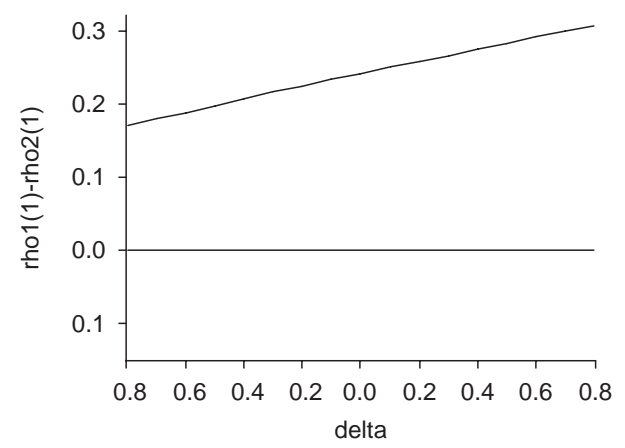

$($ phi $=0, d=0.49$, vareta $=0.1)$

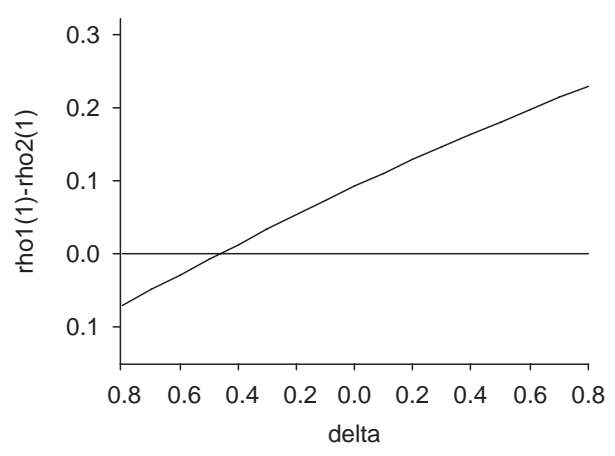

$(p h i=0.98, d=0$, vareta $=0.05)$

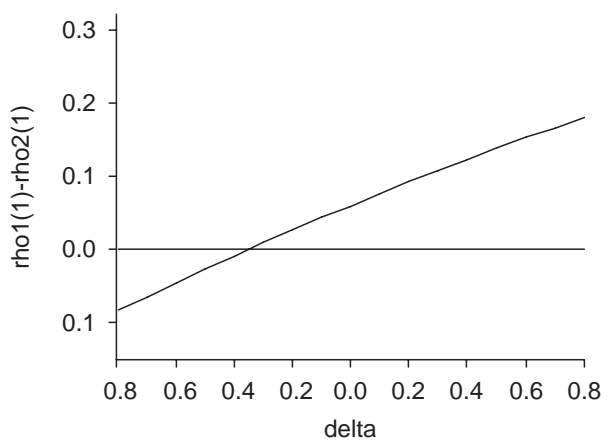

Fig. 2. Differences between the first order autocorrelations of absolute and squared returns as a function of the correlation between $\varepsilon_{t}$ and $\eta_{t+1}$ in four A-LMSV models.

negative if $\delta$ is large enough. The magnitude of this effect is larger than the corresponding effect on the autocorrelations of squared returns if $\left|\delta \sigma_{\eta} \lambda_{k}\right|<0.627$. Even more, the combination of negative correlations between $\varepsilon_{t}$ and $\eta_{t+1}$ and long-memory generates the possibility of negative autocorrelations in absolute observations. As an illustration, the left column of Fig. 1 plots the acf of absolute returns for the same models considered above. We observe that, in general, the autocorrelations decay toward zero monotonically. However, in the first model, the autocorrelations increase for the first few lags and then decay toward zero.

A property that has often interested researches dealing with models for second order moments, is the so-called Taylor effect that states that the autocorrelations of absolute returns are larger than the autocorrelations of squares. In the context of symmetric and stationary ARSV models, Mora-Galán et al. (2004) show that, if the persistence, measured by $\phi$, is large, the autocorrelations of powers of absolute observations are maximized when the power is close to one. They also show that the Taylor effect is reinforced when $\varepsilon_{t}$ has a leptokurtic distribution. The results previously described suggest that, if the correlation between the errors is negative, the Taylor effect may disappear. This result is illustrated in Fig. 2 which plots the differences $\rho_{1}(1)-\rho_{2}(1)$ as a function of $\delta$ for the same four models considered before. The first result that emerges from Fig. 2 is that there is an approximately linear positive relationship between $\delta$ and $\rho_{1}(1)-\rho_{2}(1)$. When $\delta$ is negative and large enough in magnitude, the autocorrelations of squares could be larger than the autocorrelations of absolute returns.

As we mentioned in the Introduction, real time series of financial returns are often characterized by significant autocorrelations of powers of absolute returns that decay very slowly toward zero and by the asymmetric response of volatility to positive and negative returns. On top of this, these series usually have excess kurtosis and the autocorrelations of squares are rather small in magnitude. We now analyze whether the proposed A-LMSV is able to explain simultaneously the excess kurtosis and small autocorrelations of squares. We have seen before that the presence of leverage effect does not have any effect on the kurtosis and only very marginal effects on the autocorrelations of squares. Therefore, whether the parameter $\delta$ is zero or not is not going to change the ability of the A-LMSV model to 


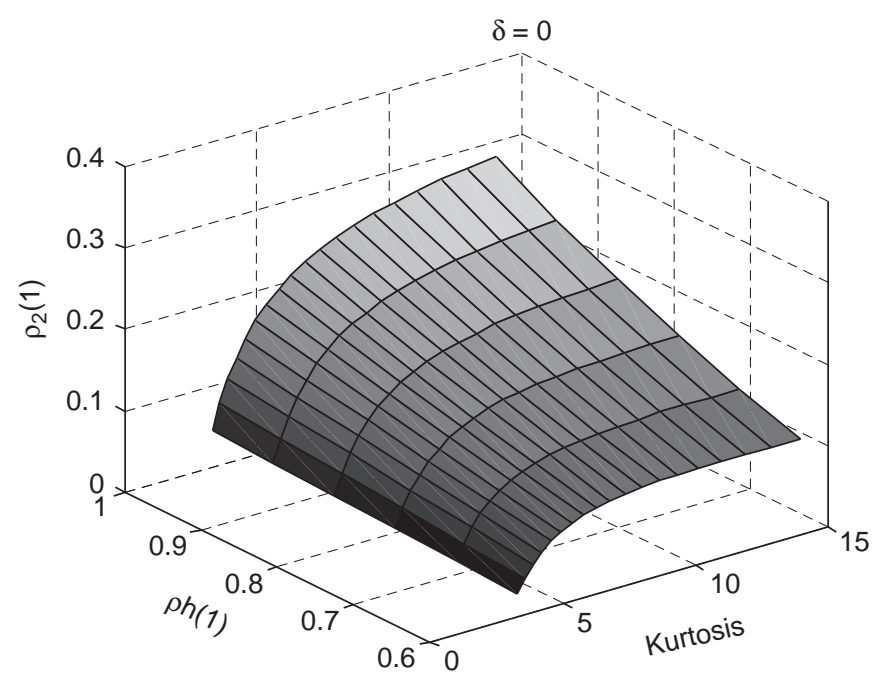

Fig. 3. Relationship between kurtosis, first order autocorrelation of squared observations, $\rho_{2}(1)$ and the first order autocorrelation of the underlying volatility $\rho_{h}(1)$, for A-LMSV models with $\delta=0$.

represent simultaneously both effects. However, we want to analyze how the presence of long-memory may change the relationship between kurtosis, $\kappa_{y}$, and $\rho_{2}(1)$ which is given by

$$
\rho_{2}(1)=\frac{\left(\frac{k_{y}}{3}\right)^{\rho_{h}(1)}\left(1+\left(\delta \sigma_{\eta}\right)^{2}\right)-1}{k_{y}-1} .
$$

Fig. 3 plots the relationship between $\kappa_{y}$ and $\rho_{2}(1)$ as a function of the autocorrelation of order 1 of the underlying $\log$-volatility, $\rho_{h}(1)$, when $\delta=0$. Given that neither the kurtosis nor the autocorrelations of squares depend on the asymmetry parameter, the results for other values of $\delta$ are similar to the ones plotted in Fig. 3. Note that $\rho_{h}(1)$ depends on $\phi$ and $d$. For fixed $d, \rho_{h}(1)$ is a non-monotonous function of $\phi$. On the other hand, when $\phi$ is fixed, the autocorrelations of the log-volatility increase with the long-memory parameter, $d$. Therefore, when interpreting Fig. 3, if we assume that $\phi$ is fixed, larger values of $\rho_{h}(1)$ are identified with larger values of $d$. We can observe that for a given kurtosis, $\kappa_{y}$, the autocorrelation of order one of squares increases with the long-memory parameter, $d$. However, the rate of growth of $\rho_{2}(1)$ is very small for low values of the kurtosis and increases with it. On the other hand, given $\rho_{2}(1)$, the kurtosis decreases as $d$ increases. Fig. 3 also illustrates that there is a wide range of combinations of the parameters that govern the dynamic evolution of the underlying volatilities, $\phi$ and $d$ able to generate series with large kurtosis and small autocorrelations of squares as the ones usually observed in real time series.

The asymmetric response of volatility to positive and negative returns is reflected in the cross-correlations between $y_{t}$ and $\left|y_{t+k}\right|^{c}$ for $c=1$ and 2. If the distribution of $y_{t}$ is symmetric, the main difference between heteroscedastic series with and without leverage effect is that, in the latter case the correlations between returns and future powers of absolute returns is zero while in the former they are different from zero. Consequently, another instrument for the identification of the leverage effect is the correlation between returns and future absolute returns to the power $c$. We consider the more interesting cases from the empirical point of view of $c=1$ and 2. In these cases, the covariances are given by

$$
\operatorname{Cov}\left(y_{t},\left|y_{t+k}\right|^{c}\right)=\left\{\begin{array}{cl}
0.5 \sigma_{*}^{2} \sqrt{\frac{2}{\pi}} \delta \sigma_{\eta} \lambda_{k} \exp \left\{\frac{\sigma_{h}^{2}}{4}\left(\rho_{h}(k)+1\right)\right\}, & c=1, \\
\sigma_{*}^{3} \delta \sigma_{\eta} \lambda_{k} \exp \left\{\frac{\sigma_{h}^{2}}{8}\left(4 \rho_{h}(k)+5\right)\right\}, & c=2 .
\end{array}\right.
$$

Note that the expression of the covariance between returns and squared returns has been derived by Taylor (2005) in the short-memory case. However, his value of $\operatorname{Cov}\left(y_{t},\left|y_{t+k}\right|^{2}\right)$ is twice the value obtained from (7) with $c=2$ and 
$d=0$. On the other hand, the variance of $\left|y_{t}\right|^{c}$, derived by Harvey (1998) for the symmetric LMSV model with Gaussian errors, is given by

$$
\operatorname{Var}\left(\left|y_{t}\right|^{c}\right)=\sigma_{*}^{2 c} 2^{c} \exp \left(c^{2} \frac{\sigma_{h}^{2}}{2}\right)\left(\frac{\Gamma\left(c+\frac{1}{2}\right)}{\Gamma\left(\frac{1}{2}\right)}-\left[\frac{\Gamma\left(\frac{c}{2}+\frac{1}{2}\right)}{\Gamma\left(\frac{1}{2}\right)}\right]^{2}\right) .
$$

Given that the asymmetry does not change the marginal moments of $y_{t}$ and given the expression of the variance of $y_{t}$ in (3), it is possible to derive the following expression for the correlation between $y_{t}$ and $\left|y_{t+k}\right|^{c}$

$$
\operatorname{Corr}\left(y_{t},\left|y_{t+k}\right|^{c}\right)= \begin{cases}\frac{0.5 \delta \sigma_{\eta} \lambda_{k} \exp \left\{0.25 \sigma_{h}^{2} \rho_{h}(k)\right\}}{\exp \left\{\frac{\sigma_{h}^{2}}{8}\right\} \sqrt{\frac{\pi}{2} \exp \left\{0.25 \sigma_{h}^{2}\right\}-1}}, & c=1, \\ \frac{\delta \sigma_{\eta} \lambda_{k} \exp \left\{0.5 \sigma_{h}^{2} \rho_{h}(k)\right\}}{\exp \left\{\frac{\sigma_{h}^{2}}{8}\right\} \sqrt{3 \exp \left\{\sigma_{h}^{2}\right\}-1}}, & c=2 .\end{cases}
$$

From (9), it is clear that the correlations between $y_{t}$ and $\left|y_{t+k}\right|^{c}$ have the same sign as $\delta$ and that their absolute values increase with the absolute value of $\delta$. As an illustration, Fig. 4 plots these correlations for the same models considered above. It is possible to observe that the correlations are only slightly larger in absolute value between observations and future squares than between observations and future absolute values in the model with $\phi=0.5, d=0.49$ and $\sigma_{\eta}^{2}=0.1$. In all the other models, they are very similar. It is also interesting to observe that the cross-correlations plotted in Fig. 4 show the same pattern as the corresponding autocorrelations in the sense that they decay toward zero hyperbolically when the volatility has long-memory while the decay is exponential in the short-memory model.

\section{Properties of the FIEGARCH model}

The FIEGARCH model was proposed by Bollerslev and Mikkelsen (1996). In its simplest form, the FIEGARCH $(1, d, 0)$ model is given by

$$
\begin{aligned}
& y_{t}=\sigma_{t} \varepsilon_{t}, \\
& (1-\phi L)(1-L)^{d} \log \sigma_{t}^{2}=\omega+g\left(\varepsilon_{t-1}\right),
\end{aligned}
$$

where $g\left(\varepsilon_{t}\right)=\alpha\left(\left|\varepsilon_{t}\right|-\sqrt{\frac{2}{\pi}}\right)+\gamma \varepsilon_{t}$ and $\varepsilon_{t}$ is a Gaussian white noise with variance 1 . The parameter $\gamma$ measures the leverage effect while, as before, $d$ is the long-memory parameter. When $d=0$, the short-memory EGARCH model of Nelson (1991) is obtained. Note that the main difference between the A-LMSV model and the FIEGARCH model is the way the noise is defined in the log-volatility equation; see Zaffaroni (2005) who proposes an exponential specification of the volatility that encompasses both models. The FIEGARCH model is stationary if $|\phi|<1$ and $|d|<0.5$. He et al. (2002) have derived the kurtosis and autocorrelations of absolute and squared observations for the shortmemory EGARCH model, i.e. model (10) with $d=0$. Following their arguments, we have obtained the kurtosis and acf of $\left|y_{t}\right|^{c}$ for $c=1$ and 2 in the long-memory FIEGARCH model. In particular, if the stationarity conditions are satisfied, the kurtosis is given by

$$
k_{y}=3 \frac{\prod_{j=1}^{\infty} E\left\{\exp \left[2 \lambda_{j} g\right]\right\}}{\left\{\prod_{j=1}^{\infty} E\left(\lambda_{j} g\right)\right\}^{2}},
$$

where for notational simplicity $g=g\left(\varepsilon_{t}\right)$ and $\lambda_{j}$ is defined as in (5). Furthermore, if the errors are normally distributed, the expectations involved in equation (11) can be evaluated using the following result due to Nelson (1991):

$$
E[\exp (b g)]=\left\{\Phi\left(b c_{1}\right) \exp \left\{0.5 b^{2} c_{1}^{2}\right\}+\Phi\left(b c_{2}\right) \exp \left\{0.5 b^{2} c_{2}^{2}\right\}\right\} \exp \left\{-b \alpha(2 / \pi)^{1 / 2}\right\},
$$


$(p h i=0, d=0.49$, vareta $=0.05)$

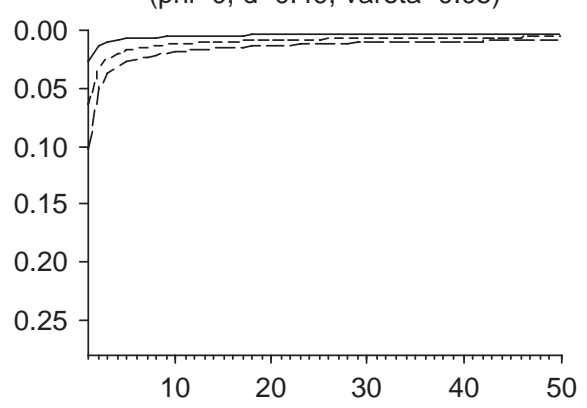

$(p h i=0, d=0.49$, vareta $=0.1)$

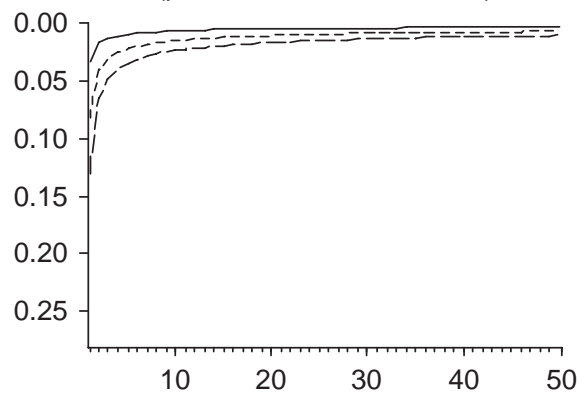

(phi=0.5, d=0.49, vareta $=0.1$ )

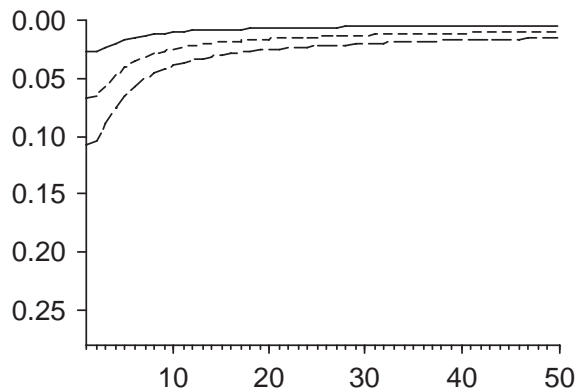

$(p h i=0.98, d=0$, vareta $=0.05)$

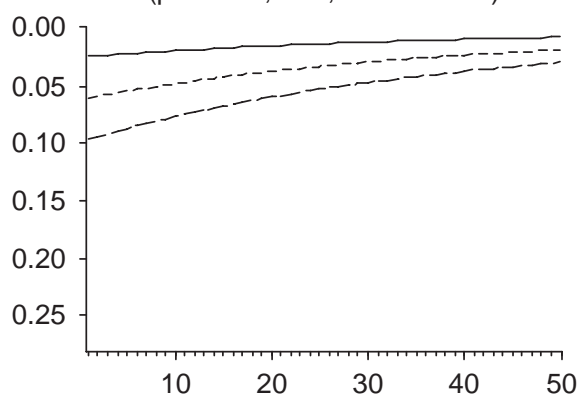

(phi=0, d=0.49, vareta $=0.05$ )

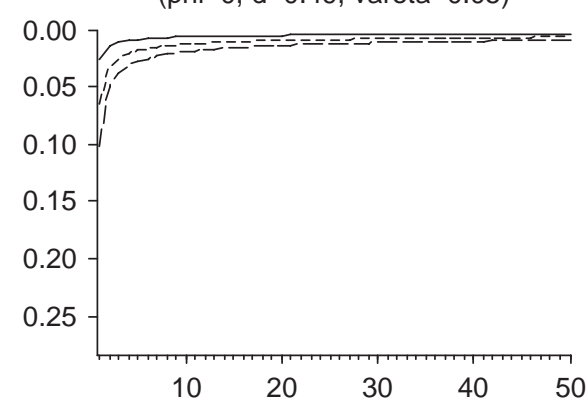

$($ phi $=0, d=0.49$, vareta $=0.1$ )

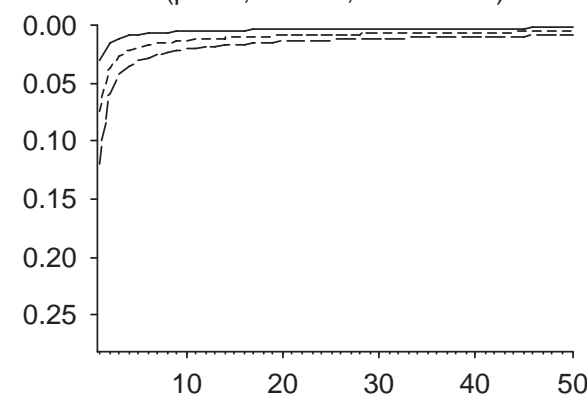

$(p h i=0.5, d=0.49$, vareta $=0.1)$

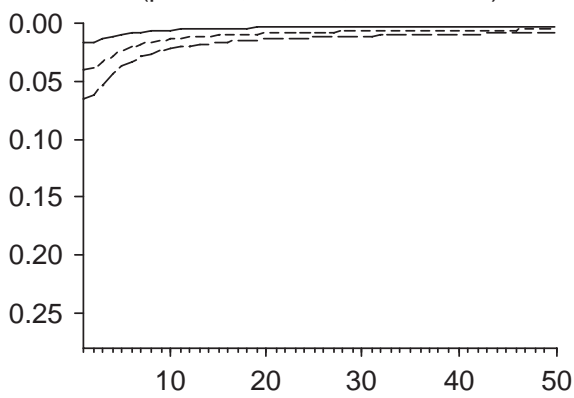

(phi $=0.98, d=0$, vareta $=0.05$ )

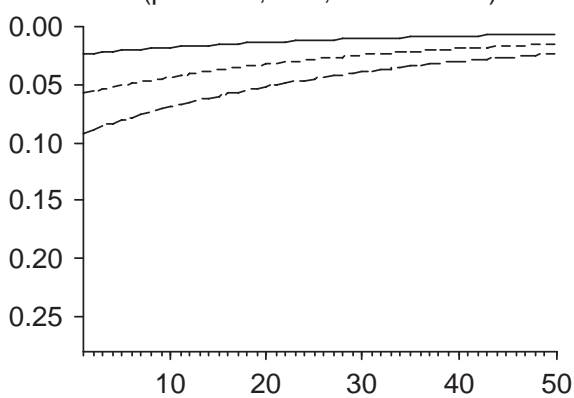

Fig. 4. Correlations between $y_{t}$ and $\left|y_{t+k}\right|$ (left column) and $y_{t}$ and $y_{t+k}^{2}$ (right column) in four A-LMSV models with different values of $\delta$ : continuous $(\delta=-0.2)$, dotted $(\delta=-0.5)$ and dashed $(\delta=-0.8)$.

where $c_{1}=\alpha+\gamma$ and $c_{2}=\alpha-\gamma$. Although it is not evident from expression (11), given the parameters $\alpha, \phi$ and $d$, the kurtosis of $y_{t}$ increases as the magnitude of the asymmetry parameter increases. This is an important difference with respect to the A-LMSV model in which we have seen that the kurtosis does not depend on the leverage effect. 
On the other hand, the acf of $\left|y_{t}\right|^{c}$ in the FIEGARCH model is given by

$$
\rho_{c}(k)=\frac{E\left[\left|\varepsilon_{t}\right|^{c} \exp \left(0.5 c \lambda_{k} g\right)\right]}{\kappa_{c} \prod_{j=1}^{\infty} E\left[\exp \left(c \lambda_{j} g\right)\right]-P_{3}}\left[\left(\frac{\pi}{2}\right)^{(2-c) / 2} P_{1} P_{2}-P_{3}\right],
$$

where $P_{1}=\prod_{j=1}^{k} E\left[\exp \left(0.5 c \lambda_{j} g\right)\right], P_{2}=\prod_{j=1}^{\infty} E\left[\exp \left(0.5 c\left(\lambda_{j+1+k}+\lambda_{j}\right)\right) g\right]$ and $P_{3}=\prod_{j=1}^{\infty}\left(E\left[\exp \left(0.5 c \lambda_{j} g\right)\right]\right)^{2}$ and $\lambda_{k}$ is defined as in A-LMSV model. Furthermore, He et al. (2002) show that

$$
\begin{aligned}
E\left[\left|\varepsilon_{t}\right|^{c} \exp (b g)\right]= & (2 \pi)^{-1 / 2} \Gamma(c+1) \exp \left\{-b \alpha(2 \pi)^{1 / 2}\right\} D_{(-(c+1))}(-b(\alpha+\gamma)) \\
& +\exp \left\{-b^{2} \alpha \gamma\right\} D_{(-(c+1))}(-b(\alpha-\gamma))
\end{aligned}
$$

where $D_{(q)}$ is the parabolic cylinder function and $\Phi(\cdot)$ is the standard normal cumulative function. Nelson (1991) derived this expression for squares, i.e. $c=2$. First, note that the autocorrelations of squares and absolute returns are symmetric with respect to the asymmetry parameter, $\gamma$.

As an illustration, Fig. 5 plots the acf of absolute and squared returns for the following FIEGARCH models: $\{\phi=0, d=0.49, \alpha=0.2\},\{\phi=0.5, d=0.49, \alpha=0.1\},\{\phi=0.5, d=0.49, \alpha=0.2\}$ and $\{\phi=0.5, d=0, \alpha=0.2\}$ with the asymmetry parameter $\gamma=0,-0.1,-0.2$, and -0.3 . These models have been chosen to resemble the parameter values often estimated when the FIEGARCH model is fitted to real time series; see, for example, Bollerslev and Mikkelsen (1996, 1999). Comparing the autocorrelations of the FIEGARCH model with the corresponding autocorrelations of the A-LMSV model plotted in Fig. 1, we can observe that while in the latter model, the autocorrelations of squares are nearly the same regardless of the asymmetry parameter, they may be different in the FIEGARCH model. In general, the first order autocorrelations of squares increase with the magnitude of $\gamma$. Furthermore, note that, in the considered models, the autocorrelations of squares and absolute observations are rather similar between them when the asymmetry parameter is small. However, the Taylor effect is reinforced by the leverage effect in the models with both parameters $\phi$ and $d$ different from zero while it seems that it is not a property of the short-memory model and of the model with $\phi=0$. To have a clearer picture of this phenomena, Fig. 6 plots the differences between the first order autocorrelations of absolute and squared returns for the same four FIEGARCH models described above. This figure confirms the suspicion that when $\phi=0$ or $d=0$ there is not Taylor effect. On the other hand, in the models with both $\phi$ and $d$ different from zero, it is present if $\gamma$ is relatively large in absolute value. Comparing Figs. 6 and 2, we can observe that there are clear differences with respect to the Taylor effect in the A-LMSV and FIEGARCH models. When looking at FIEGARCH models, the differences between the first order autocorrelations of absolute values and those of squares are not an increasing function of the asymmetry parameter. On the other hand, there are particular specifications of the FIEGARCH model in which the Taylor effect is not a property unless the magnitude of the asymmetry parameter is very large.

Given that it is rather difficult to find an analytical expression relating the kurtosis and the autocorrelation of order one of squared observations, Fig. 7 plots this relationship for different FIEGARCH models. This figure illustrates that as the kurtosis increases (the asymmetry increases), the autocorrelation of order one of squares also increases. On the other hand, given $\phi, \alpha$ and $\gamma$, larger values of the long-memory parameter, $d$, imply smaller autocorrelations of squares except when the kurtosis (the asymmetry) is very large. Carnero et al. (2004) have also shown for the short-memory EGARCH model that, given the kurtosis, the autocorrelation of order one of squared observations decreases with the persistence parameter, $\phi$. On the other hand, for fixed $\phi, d$ and $\gamma$, the autocorrelations of squares are larger the larger is the ARCH effect, i.e. $\alpha$. Once more, this relationship can be reverse for large values of $\gamma$. In any case, it is important to point out that the effect of $d$ and $\alpha$ on $\rho_{2}(1)$ is relatively weak while the effect of the asymmetry parameter, $\gamma$, is rather strong.

Comparing now the results illustrated in Fig. 7 with the corresponding results for the A-LMSV model illustrated in Fig. 3, we can observe that in both models, the autocorrelations of order 1 of squares increase with the kurtosis. However, while in the A-LMSV model, the kurtosis is the same regardless the asymmetry parameter, in the FIEGARCH model, the kurtosis depends on the asymmetry. Therefore, given the parameters that measure the evolution of volatility, $\alpha$ in the FIEGARCH model and $\sigma_{\eta}^{2}$ in the A-LMSV models, respectively, its persistence, $\phi$, and its memory, $d$, the kurtosis and the autocorrelations of squares increase with the leverage effect in the FIEGARCH model while they are approximately constant in the A-LMSV model. On the other hand, for fixed $\alpha$ and $\sigma_{\eta}^{2}$, the autocorrelations of squares 
(phi=0, $d=0.49$, alpha $=0.2)$

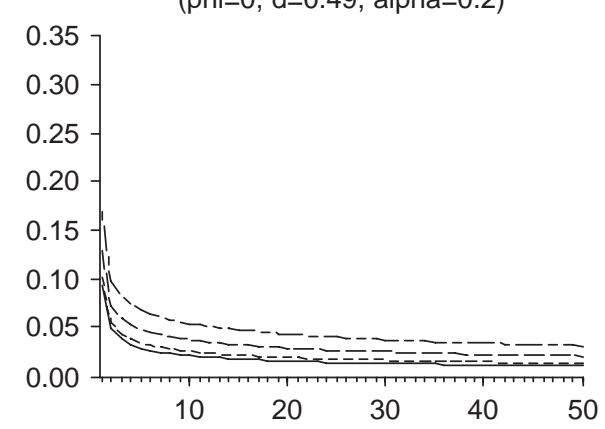

(phi=0.5, $d=0.49$, alpha $=0.1$ )

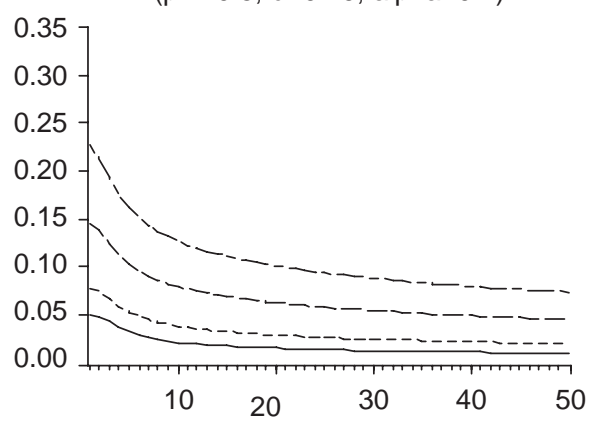

(phi=0.5, $d=0.49$, alpha $=0.2$ )

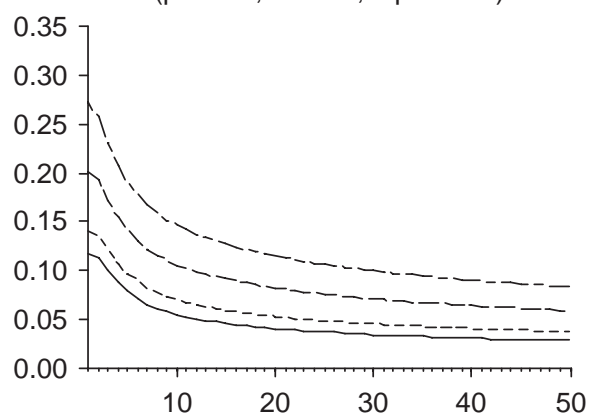

(phi $=0.5, d=0$, alpha $=0.2$ )

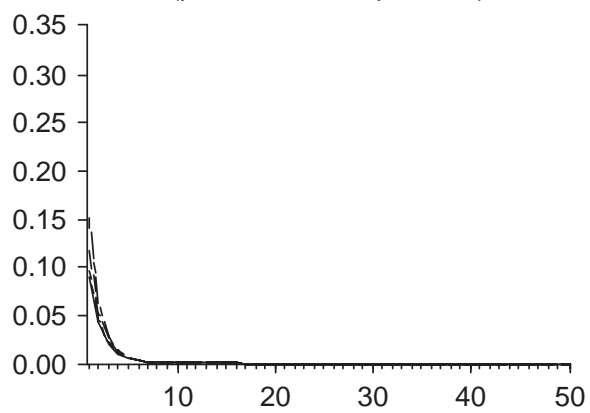

(phi $=0, d=0.49$, alpha $=0.2$ )

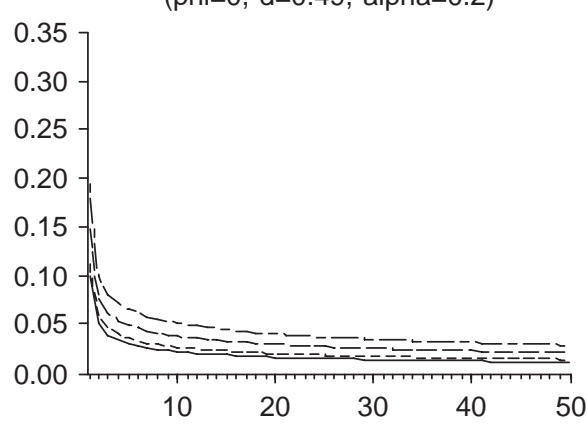

(phi=0.5, d=0.49, alpha=0.1)

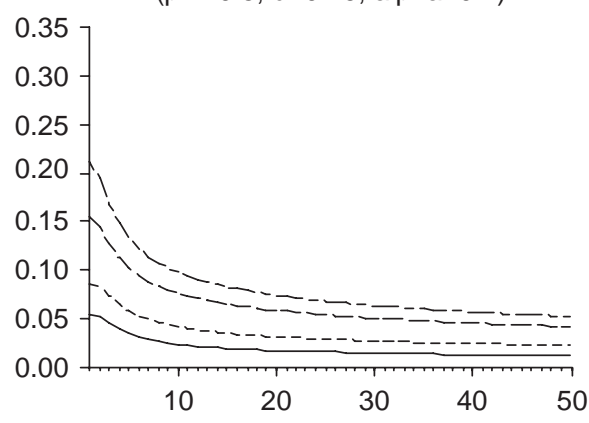

(phi=0.5, $d=0.49$, alpha $=0.2$ )

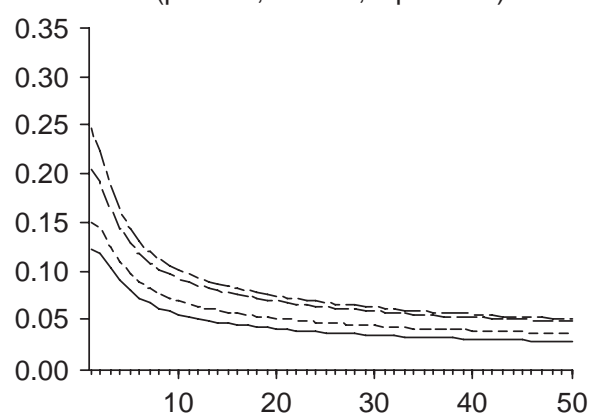

(phi=0.5, d=0, alpha $=0.2)$

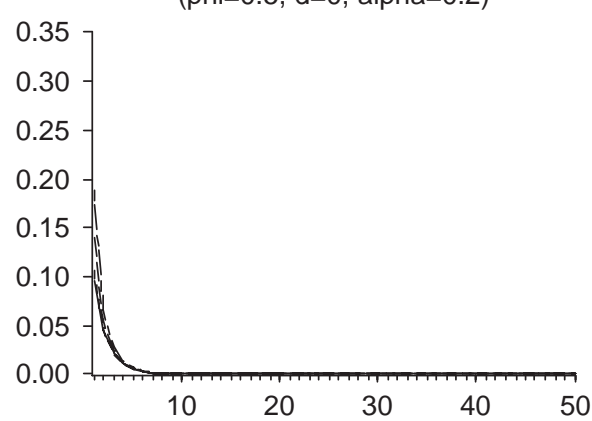

Fig. 5. Auto correlations of $\left|y_{t}\right|$ (left column) and $y_{t}^{2}$ (right column) in four FIEGARCH models with different values of $\gamma$ : continuous $(\gamma=0)$, dotted $(\gamma=-0.1)$, dashed $(\gamma=-0.2)$ and dotted-dashed $(\gamma=-0.3)$.

increase with $\phi$ and $d$ in the A-LMSV model while they decrease in the FIEGARCH model. In both models it is possible to observe that the variations in the values of $\rho_{2}(1)$ are small for moderate values of the kurtosis while they are large when the kurtosis is large. 

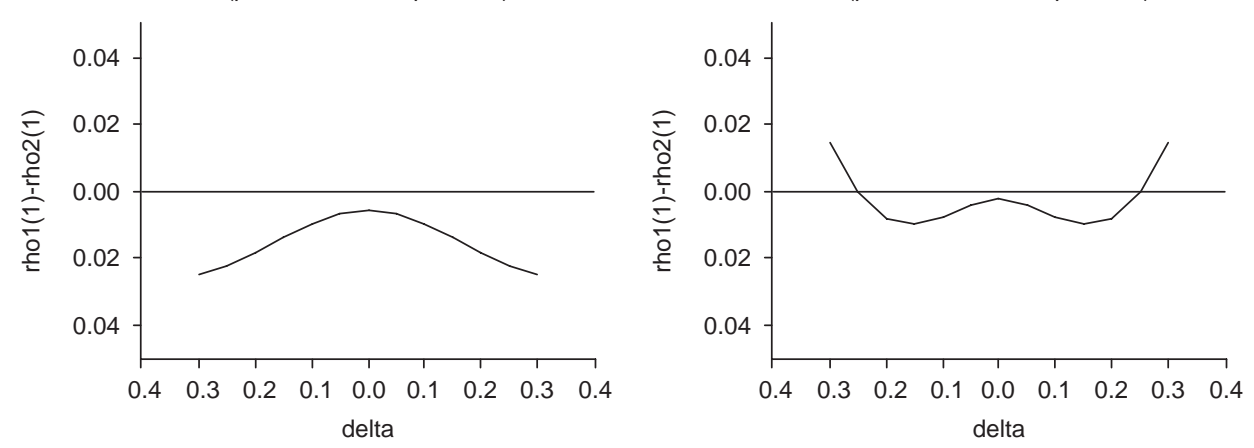

$($ phi $=0.5, \mathrm{~d}=0.49$, alpha $=0.2)$

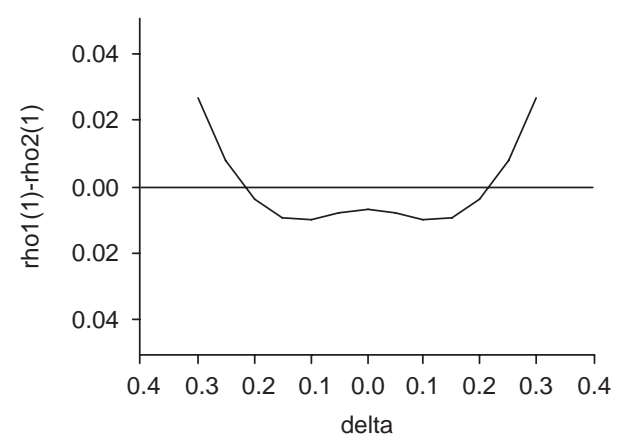

(phi=0.5, d=0, alpha=0.2)

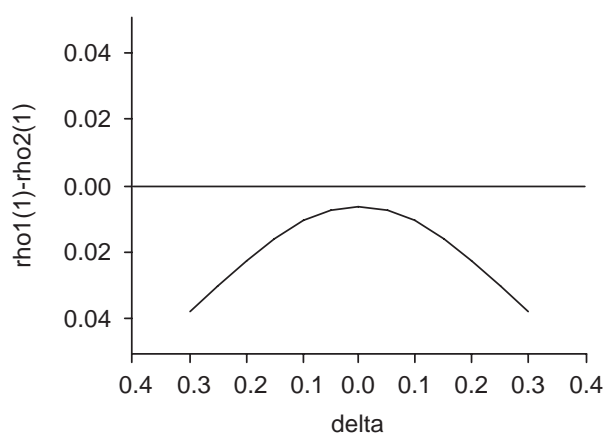

Fig. 6. Differences between the first order autocorrelations of absolute and squared returns as a function of $\gamma$ in FIEGARCH models.

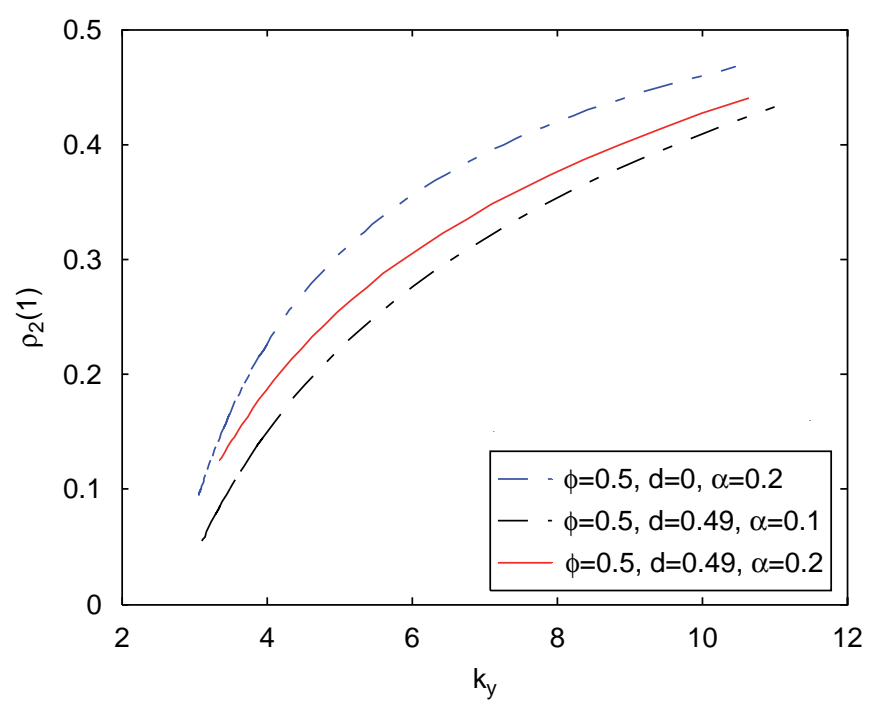

Fig. 7. Relationship between kurtosis and first order autocorrelation of squared observations for different values of the persistence for FIEGARCH $(1, d, 0)$ models.

As in the A-LMSV model, we have also derived the cross-correlations between returns and powers of absolute returns. In particular,

$$
\begin{aligned}
& \operatorname{Corr}\left(y_{t},\left|y_{t+k}\right|^{c}\right) \\
& =\frac{E\left[\exp \left(0.5 c \lambda_{k} g\left(\varepsilon_{t}\right)\right) \varepsilon_{t}\right] \prod_{j=1}^{k-1} \exp \left[E\left[0.5 c \lambda_{j} g\right]\right] \prod_{j=1}^{\infty} E\left[\exp \left(0.5\left(c \lambda_{j+k}+\lambda_{j}\right)\right) g\right]}{\left[\kappa_{c} \prod_{j=1}^{\infty} E\left[\exp \left(c \lambda_{j} g\right)\right]-\left[\prod_{j=1}^{\infty} E\left[\exp \left(0.5 c \lambda_{j} g\right)\right]\right]^{c}\right]^{1 / 2}\left[\prod_{j=1}^{\infty} E\left[\exp \left(\lambda_{j} g\right)\right]\right]^{1 / 2}} .
\end{aligned}
$$



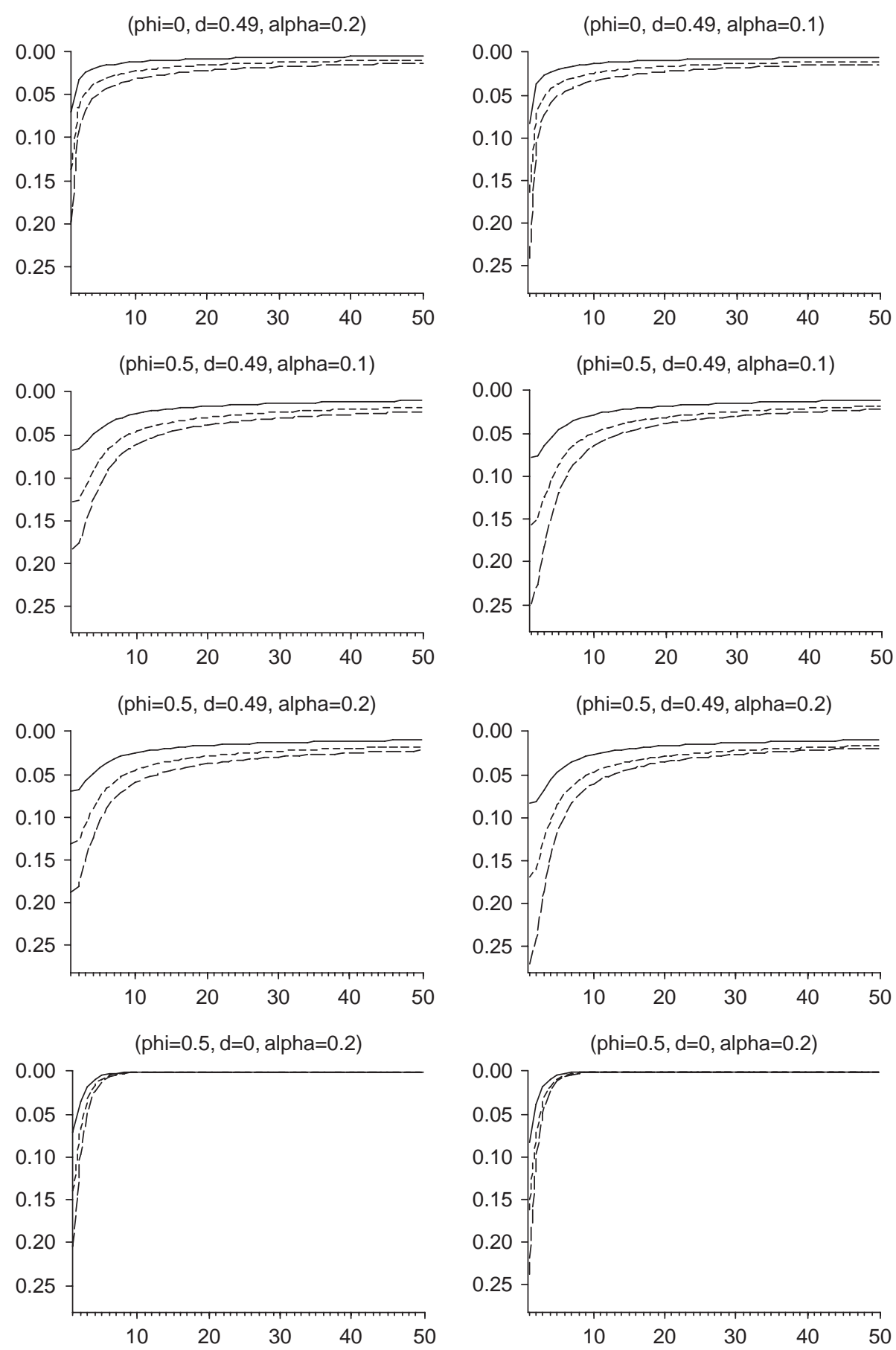

Fig. 8. Correlations between $y_{t}$ and $\left|y_{t+k}\right|$ (left column) and $y_{t}$ and $y_{t+k}^{2}$ (right column) in four FIEGARCH models with different values of $\gamma$ : continuous $(\gamma=-0.1)$, dotted $(\gamma=-0.2)$ and dashed $(\gamma=-0.3)$.

The cross-correlations in expression (13) are symmetric with respect to the parameter $\gamma$. Once more, we illustrate their shape by plotting them in Fig. 8 for the same four models considered before and $c=1,2$. As in the A-LMSV model, we can observe that the cross-correlations between $y_{t}$ and $y_{t+k}^{2}$ are larger in magnitude than between $y_{t}$ and $\left|y_{t+k}\right|$. Furthermore, comparing these cross-correlations with the ones plotted in Fig. 4 for the A-LMSV models, it 
is possible to observe that, in general, the FIEGARCH model generates cross-correlations which are larger for small lags and then decay toward zero very quickly. Note that we have chosen the FIEGARCH models so that the first order autocorrelations of squares and absolute values are similar. Obviously, one can chose to fit the first order crosscorrelations in both models and, in this case, the autocorrelations of squares and absolute values of the FIEGARCH model will be, in general, larger than those of the A-LMSV model.

\section{Empirical illustration}

In this section we evaluate the performance of the A-LMSV $(1, d, 0)$ and $\operatorname{FIEGARCH}(1, d, 0)$ models in capturing the empirical features of financial data. For this purpose, we analyze daily close prices of the S\&P 500 and DAX composite indexes observed from November 26, 1990 to September 6, 2006, respectively. The sample size is 3980 observations in each case. The series of returns have been computed as usual, $y_{t}=100\left(\log p_{t}-\log p_{t-1}\right)$, where $p_{t}$ denotes the price at time $t$.

The series of S\&P500 prices and returns have been plotted in Fig. 9. The returns have a kurtosis of 4.053 and show volatility clustering. The presence of conditional heteroscedasticity can also be observed in the correlograms of the absolute and squared observations also plotted in Fig. 9. In both correlograms, the sample autocorrelations are significant and very persistent. The observed decay of the correlations is not compatible with the expected decay if squared and absolute returns could be represented by a short-memory model. In consequence, it seems that the volatility of the S\&P500 returns should be approximated by a conditional heteroscedasticity model with long-memory. Fig. 9 also plots the $\operatorname{Corr}\left(y_{t},\left|y_{t+k}\right|\right)$ and $\operatorname{Corr}\left(y_{t}, y_{t+k}^{2}\right)$. In both cases, the cross-correlations are similar in magnitude, being negative and significant. Therefore, it seems that a model with leverage effect could be adequate. We fit the A-LMSV and the FIEGARCH models to the series of S\&P500 returns.

The A-LMSV model has been estimated by the EMM estimator; see Gallant et al. (1997). Alternatively, Zaffaroni (2005) has proposed a Whittle estimator of A-LMSV model. However, the parameters $\sigma_{*}$ and $\delta$ are not identified by the Whittle estimator that is based on the linearizing transformation $\log \left(y_{t}^{2}\right)$. Furthermore, it is not obvious how to obtain expressions of the asymptotic variances of the estimator using the expressions given by Zaffaroni (2005). On the other hand, there are several estimators proposed to estimate separately asymmetric SV models or long-memory SV models; see, for example, Omori and Watanabe (2007) and Arteche (2006), respectively, for some very recent references. However, none of these estimators have been considered for the estimation of models with the simultaneous presence of long-memory and leverage effect. Consequently, we implement the EMM estimator with a score generator which is a GARCH model with fully nonlinear nonparametric error density. The estimated model is given by

$$
(1-\underset{(0.141)}{0.442} L)(1-L)^{0.541} \operatorname{(0.007)} \log \sigma_{t}^{2}=\eta_{t},
$$

where the scale parameter estimate is $\widehat{\sigma}_{*}=0.801$, the variance of the log-volatility noise estimate is $\widehat{\sigma}_{\eta}^{2}=0.048$ and the correlation between the level and volatility noises estimate is $\widehat{\delta}=-0.75$.

The FIEGARCH model has been estimated by QML using the GARCH package version 4.0 of Laurent and Peters (2005) with the following results:

$$
(1-\underset{(0.145)}{0.538} L)(1-L)^{0.538} \operatorname{(0.053)} \log \sigma_{t}^{2}=\underset{(0.215)}{0.453}+\underset{(0.029)}{0.143}\left(\left|\varepsilon_{t-1}\right|-\sqrt{\frac{2}{\pi}}\right)-\underset{(0.028)}{0.108} \varepsilon_{t-1},
$$

where the quantities in parenthesis are estimated standard deviations.

Note that both models have estimates of the long-memory parameter larger than 0.5 implying that the series of S\&P500 returns is not stationary. Similar results have been found by other authors analyzing different sample periods; see, for example, Bollerslev and Mikkelsen (1996) and Granger and Hyung (2004). In these circumstances, the moments implied by the estimated models are not defined. Therefore, it is not possible to compare the sample moments of the S\&P500 returns with the moments implied by each of the estimated models.

The DAX prices and returns have been plotted in Fig. 10. To avoid the pernicious effect of large extreme observations on the sample moments and estimates of the parameters that govern the volatility, all the observations larger than 7 conditional standard deviations have been corrected by substituting them by their estimated conditional standard deviation; see Carnero et al. (2007) for the effects of outliers on the identification and estimation of conditional 


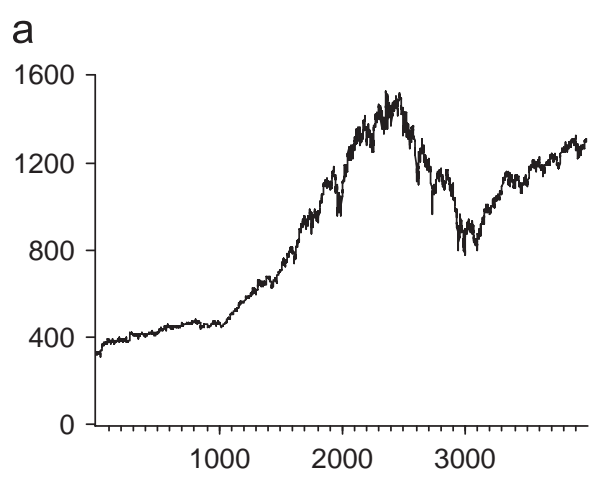

b

C
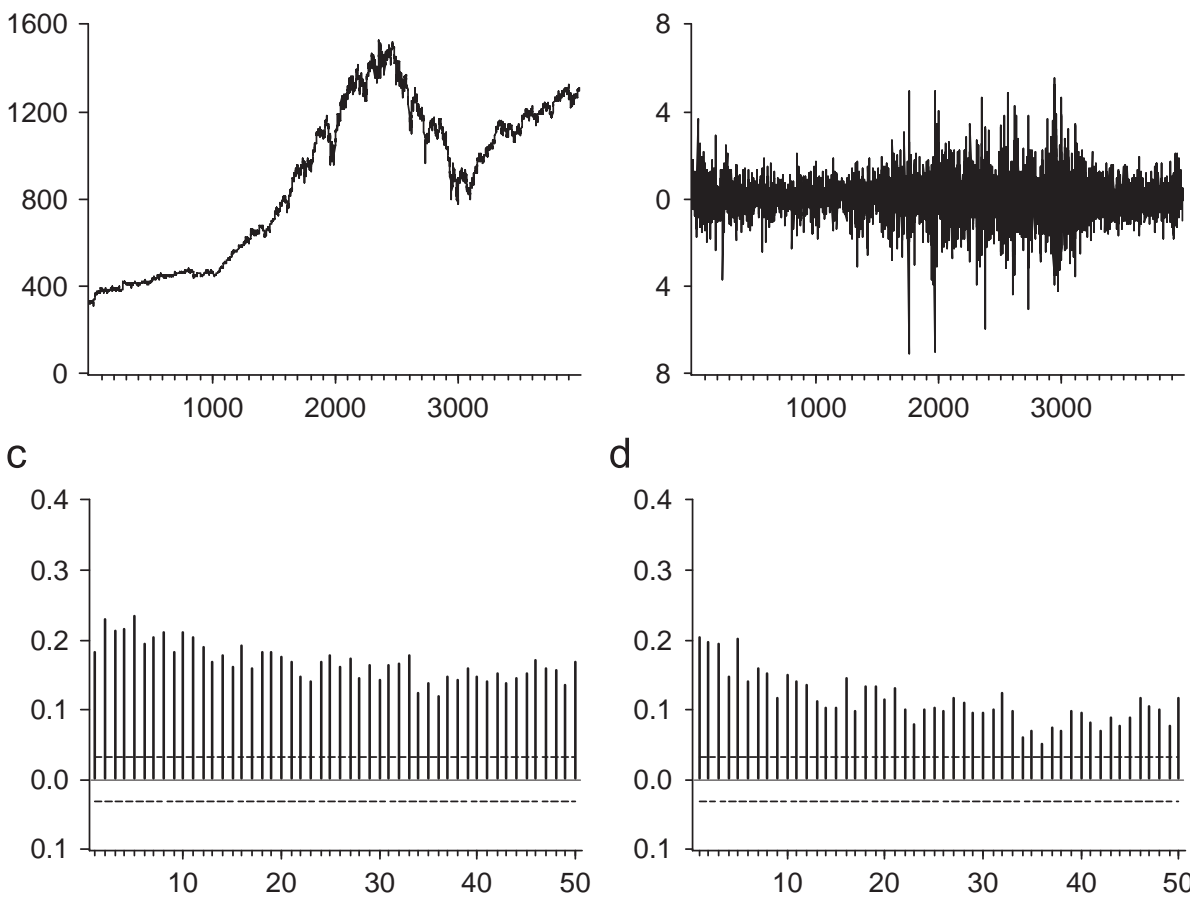

$\mathrm{d}$

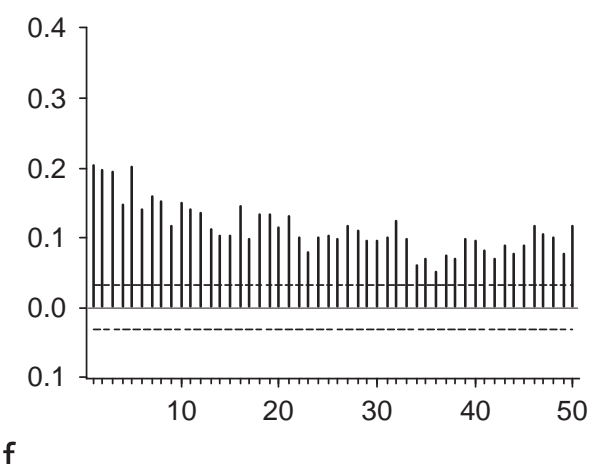

e
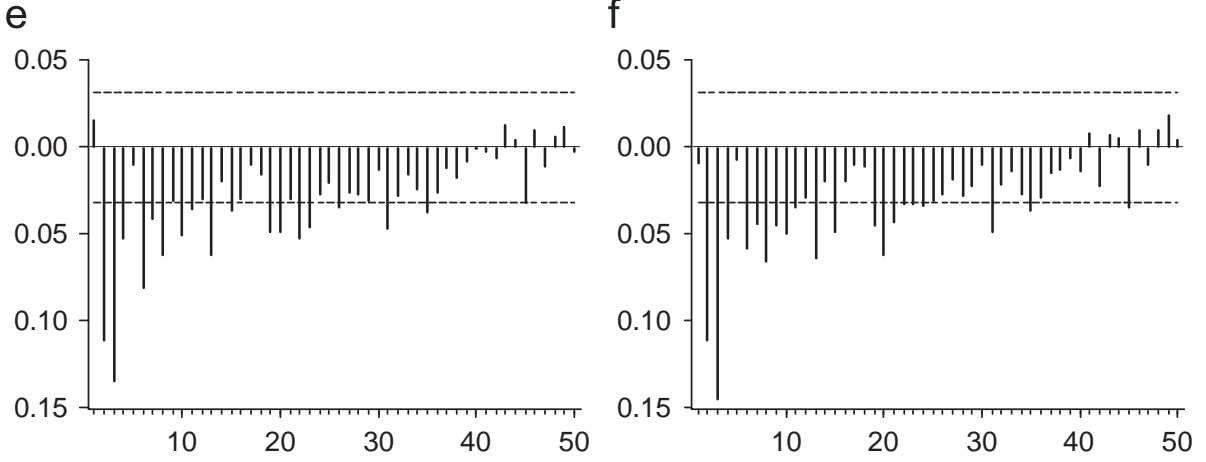

Fig. 9. (a) Observations of daily S\&P500 prices and (b) returns together with (c) sample autocorrelations of absolute returns and (d) squared returns and (e) cross-correlations between $y_{t}$ and $\left|y_{t+k}\right|$ and (f) between $y_{t}$ and $y_{t+k}^{2}$.

heteroscedasticity. In particular, the unique observation corrected corresponds to 19th August 1991. The corrected series has a kurtosis of 6.221 and also shows clear signs of conditional heteroscedasticity when looking at the correlations of squares and absolute returns plotted in Fig. 10. Both correlograms show significant positive correlations which decay slowly toward zero. Finally, the cross-correlations between $y_{t}$ and $y_{t+k}^{2}$ and between $y_{t}$ and $\left|y_{t+k}\right|$ are negative and significant. Therefore, it seems that the dynamic evolution of the DAX returns can be also represented by a conditionally heteroscedastic model with long-memory and asymmetry. As before, we fit the A-LMSV and FIGARCH models. The estimated A-LMSV model is given by

$$
(1-\underset{(0.018)}{0.883} L)(1-L)^{0.413} \operatorname{(0.008)} \log \sigma_{t}^{2}=\eta_{t}
$$

where the scale parameter estimate is $\widehat{\sigma}_{*}=0.906$, the variance of the log-volatility noise estimate is $\widehat{\sigma}_{\eta}^{2}=0.01$ and the correlation between the level and volatility noises estimate is $\widehat{\delta}=-0.373$. 
a

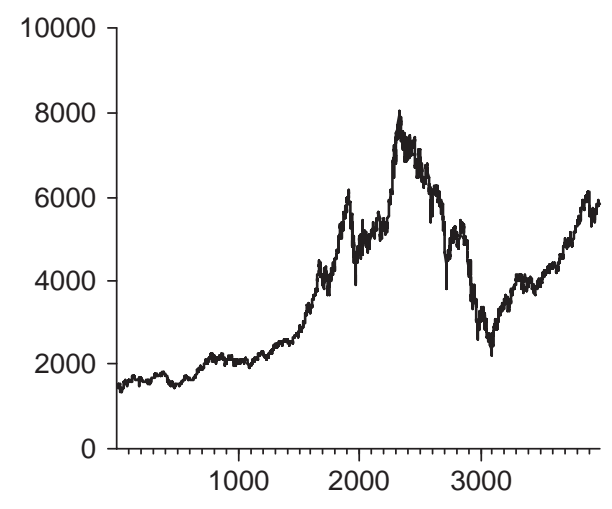

C

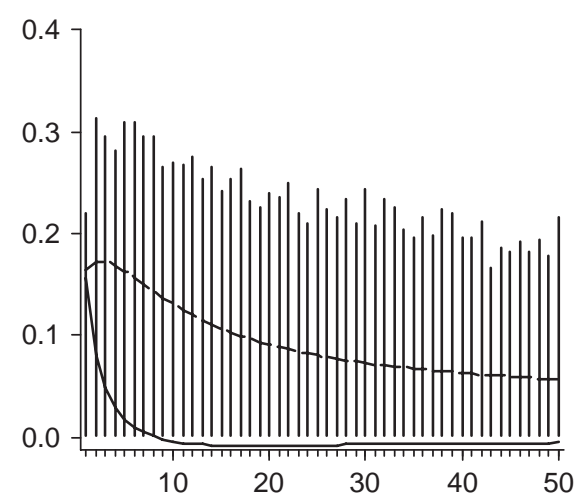

e

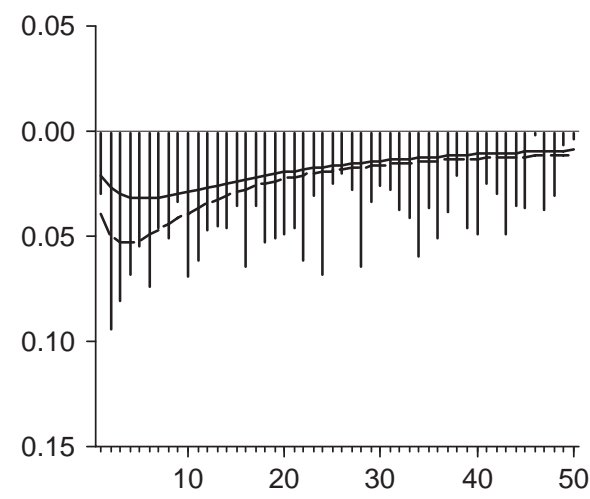

b

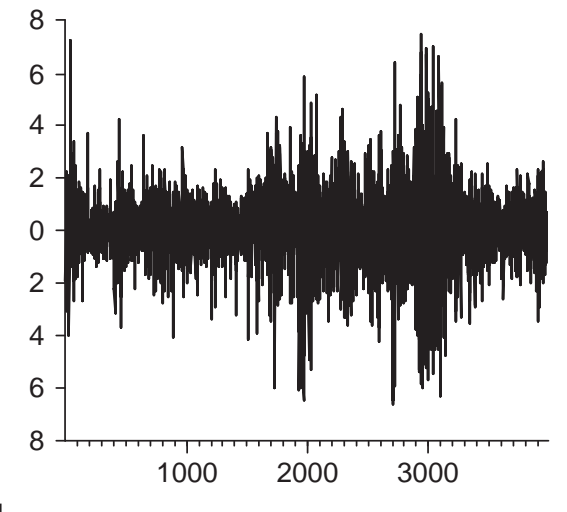

d

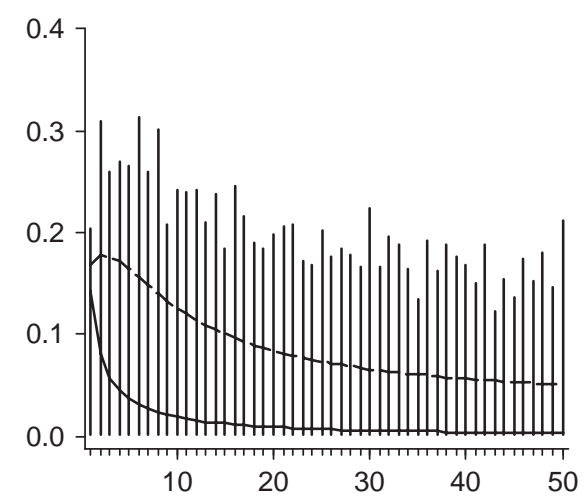

f

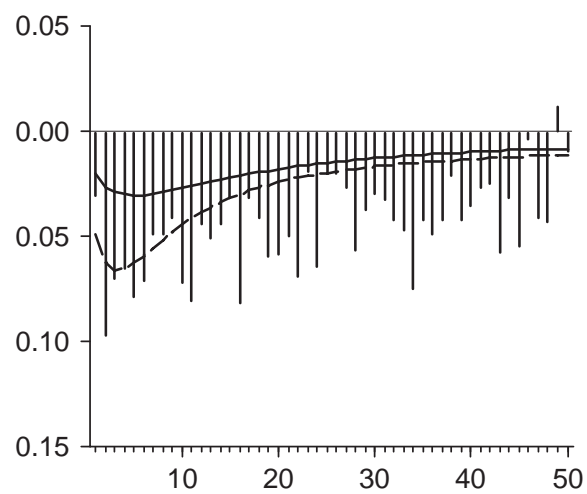

Fig. 10. (a) Observations of daily DAX prices and (b) returns together with (c) sample autocorrelations of absolute returns and (d) squared returns and (e) cross-correlations between $y_{t}$ and $\left|y_{t}\right|$ and (f) between $y_{t}$ and $y_{t}^{2}$. In each case, the continuous and dashed lines represent the plug-in autocorrelations and cross-correlations implied by the A-LMSV and FIEGARCH models, respectively.

The estimated FIEGARCH $(1, d, 0)$ model is given by

$$
(1-\underset{(0.086)}{0.817} L)(1-L)^{0.436} \operatorname{(0.10)} \log \sigma_{t}^{2}=\underset{(0.229)}{0.837}+\underset{(0.024)}{0.140}\left(\left|\varepsilon_{t-1}\right|-\sqrt{\frac{2}{\pi}}\right)-\underset{(0.014)}{0.063} \varepsilon_{t-1} .
$$

First, note that the estimated long-memory parameter, $d$, is smaller than 0.5 in both models. Therefore, DAX returns are stationary and their moments are well defined. We now analyze which model implies moments closer to the observed moments of DAX returns. The plug-in kurtosis implied by the estimated A-LMSV and FIEGARCH models are 7.702 
and 4.449, respectively. Therefore, the kurtosis of the A-LMSV is closer to the observed kurtosis. Looking at the plug-in autocorrelations of absolute and squared returns implied by each of the two models, plotted in Fig. 10, it is possible to observe that they are clearly smaller than the observed autocorrelations. However, the sample autocorrelations of the standardized residuals are not significant. We do not report the corresponding diagnostics to save space although they are available upon request. On the other hand, the plug-in cross-correlation between $y_{t}$ and $\left|y_{t+1}\right|$ implied by the A-LMSV model, -0.021 , is closer to the corresponding sample cross-correlation, -0.029 , than the one implied by the FIEGARCH model, -0.039 . The same happens with respect to the cross-correlations between $y_{t}$ and $y_{t+1}^{2}$. The sample first order cross-correlation is -0.031 while the plug-in cross-correlations implied by the A-LMSV and FIEGARCH models are -0.021 and -0.049 , respectively. However, for other lags, the plug-in cross-correlations implied by both models are, in general, under the sample cross-correlations.

\section{Conclusions}

We propose an extension of the LMSV to represent the asymmetric response of volatility to positive and negative returns. We consider the model in which the log-volatility is specified as an $\operatorname{ARFIMA}(1, d, 0)$ model. We compare the statistical properties of the new model with the $\operatorname{FIEGARCH}(1, d, 0)$ model. As a byproduct, we derive expressions of the autocorrelations of squares and absolute returns as well as cross-correlations between these transformations of returns and the original returns when they are generated by the FIEGARCH model. We show that the kurtosis and autocorrelations of squares of the A-LMSV model are not affected by the presence of the leverage effect while in the FIEGARCH model, both moments increase with the asymmetry. Furthermore, we show that the autocorrelations of squares increase with the persistence in the A-LMSV model and decrease in the FIEGARCH model. On the other hand, in the empirically relevant A-LMSV models, the correlation between the volatility and the level of returns is negative. If this is the case, we show that the autocorrelations of absolute observations decrease with respect to the autocorrelations of the corresponding symmetric model. With respect to the FIEGARCH model, the autocorrelations of absolute returns increase with the magnitude of the asymmetry regardless of its sign. Finally, we show that the patterns of the cross-correlations between $y_{t}$ and $\left|y_{t+k}\right|^{c}$ are rather similar in the two models considered. Although the moments have been derived for the A-LMSV $(1, d, 0)$ and $\operatorname{FIEGARCH}(1, d, 0)$ models, they can be easily extended to more general specifications of the dynamic dependence of volatilities. In particular, if the log-volatility is assumed to be a general ARFIMA $(p, d, q)$ process, the results in Hosking (1981) can be used to derive the corresponding moments. Similarly, the arguments in He et al. (2002) can be used to derive moments for the $\operatorname{FIEGARCH}(p, d, q)$ model.

In general, the A-LMSV model reproduces better the empirical features of financial data: volatility persistence, excess kurtosis, autocorrelations of absolute and squared returns and cross-correlations between returns and future squared returns. In fact, the FIEGARCH model needs simultaneously high values of $d$ and $\phi$, close to the level of nonstationary, and small values of $\delta$ in order to capture simultaneously persistence and small first order autocorrelation. On the other hand, this last requirement interferes with generating large kurtosis. The Gaussian FIEGARCH model is only able to reproduce high kurtosis if $\delta$ is not close to zero. Therefore, it seems to exist, for this model, a trade off among different moments. Contrarily, the A-LMSV model is able to reproduce these three features of financial data for larger combinations of the parameters. The larger flexibility of the A-LMSV model could be expected due to the added noise in the volatility equation; see Carnero et al. (2004) for the comparison between symmetric and short-memory ARSV and GARCH $(1,1)$ models.

In an empirical application to daily S\&P500 and DAX returns, we show that when both models are fitted to real data, the conclusions in terms of the stationarity of the volatility are similar. When one of the models imply stationarity, the other does and the other way round. If the estimated parameters of both models satisfy the stationarity conditions, then the kurtosis and first order cross-autocorrelations of absolute and squared returns implied by the A-LMSV model are closer to the corresponding sample moments of the real data than ones implied by the FIEGARCH model. However, in our empirical application to the DAX returns none of the implied autocorrelations explain completely the slow decay of the autocorrelations toward zero. Consequently, and given that, as we have seen in this paper, dealing with the statistical properties of the A-LMSV model is easier than when considering the properties of the FIEGARCH model, we think that the former is a model to be considered when modelling the dynamic evolution of the volatility of series with long-memory and asymmetric response to positive and negative returns. 


\section{Acknowledgments}

We are very grateful to Ana Pérez, Stephen Taylor, two anonymous referees and participants at the seminar in the Department of Economics, Universidad de Alicante, for many helpful suggestions.

\section{References}

Arteche, J., 2006. Semiparametric estimation in perturbed long memory series. Comput. Statist. Data Anal. 51, $2118-2141$.

Baillie, R.T., Bollerslev, T., Mikkelsen, H.O., 1996. Fractionally integrated generalized autoregressive conditional heteroskedasticity. J. Econometrics 74, 3-30.

Black, F., 1976. Studies of stock price volatility changes. In: Proceedings of the 1976 Meetings of the Business and Economic Statistics.American Statistical Society, pp. 177-181.

Bollerslev, T., Mikkelsen, H., 1996. Modeling and pricing long memory in stock market volatility. J. Econometrics 73 (1), $151-184$.

Bollerslev, T., Mikkelsen, H., 1999. Long-term equity anticipation securities and stock market volatility dynamics. J. Econometrics $92,75-99$.

Breidt, F.J., Crato, N., de Lima, P., 1998. The detection and estimation of long memory in stochastic volatility. J. Econometrics 83, $325-348$.

Carnero, M.A., Peña, D., Ruiz, E., 2004. Persistence and kurtosis in GARCH and stochastic volatility models. J. Financial Econometrics 2, 319-342.

Carnero, M.A., Peña, D., Ruiz, E., 2007. Effects of outliers on the identification and estimation of GARCH models. J. Time Ser. Anal. 28 (4), 471-497.

Davidson, J., 2004. Moment and memory properties of linear conditional heteroscedasticity models, and a new model. J. Business Econom. Statist. $22,16-19$.

Ding, Z., Granger, C.W.J., Engle, R.F., 1993. A long memory property of stock market returns and a new model. J. Empirical Finance 1, 83-106.

Gallant, A.R., Hsieh, D., Tauchen, G., 1997. Estimation of stochastic volatility models with diagnostics. J. Econometrics 81, $159-192$.

Ghysels, E., Harvey, A.C., Renault, E., 1996. Stochastic volatility. In: Rao, C.R., Maddala, G.S. (Eds.), Statistical Methods in Finance. North-Holland, Amsterdam, pp. 119-191.

Granger, C.W.J., Hyung, N., 2004. Occasional structural breaks and long memory with an application to the SP500 absolute stock returns. J. Empirical Finance 11, 399-421.

Harvey, A.C., 1998. Long-memory in stochastic volatility. In: Knight, J., Satchell, S.E. (Eds.), Forecasting Volatility in Financial Markets. ButterworthHeinemann, London.

Harvey, A.C., Shephard, N.G., 1996. Estimation of an asymmetric stochastic volatility model for asset returns. J. Business Econom. Statist. 14, 429-434.

He, C., Teräsvirta, T., Malmsten, H., 2002. Moment structure of a family of first-order exponential GARCH models. Econometric Theory 18, 868-885.

Hosking, J.R.M., 1981. Fractional differencing. Biometrika 68, 165-176.

Hwang, Y., 2001. Asymmetric long memory GARCH in exchange returns. Econom. Lett. 71, 1-5.

Jacquier, E., Polson, N.G., Rossi, P.E., 2004. Bayesian analysis of stochastic volatility models with fat-tails and correlated errors. J. Econometrics $122,185-212$.

Laurent, S., Peters, J.P., 2005. GARCH 4.0, Estimating and Forecasting ARCH models. Timberlake Consultants.

Lobato, I., Savin, N.E., 1998. Long memory in stock-market trading volume. J. Business Econom. Statist. 18, 410-427.

Mora-Galán, A., Pérez, A., Ruiz, E., 2004. Stochastic Volatility and the Taylor Effect. WP04-63, Statistics and Econometrics Series. Universidad Carlos III de Madrid,

Nelson, D.B., 1991. Conditional heteroskedasticity in asset returns: a new approach. Econometrica 59, 347-370.

Omori, Y., Watanabe, T., 2007. Block sampler and posterior mode estimation for asymmetric stochastic volatility models. Comput. Statist. Data Anal. 10.1016/j.csda.2007.09.001.

Pérez, A., Ruiz, E., 2001. Finite sample properties of a QML estimator of stochastic volatility models with long memory. Econom. Lett. 70, 157-164.

Ruiz, E., Pérez, A., 2003. Asymmetric long memory GARCH: a reply to Hwang's model. Econom. Lett. 78, 415-422.

So, M.K.P., Li, W.K., Lam, K., 2002. A threshold stochastic volatility model. J. Forecasting 21, 473-500.

Taylor, S.J., 1982. Financial returns modelled by the product of two stochastic processes—a study of daily sugar prices 1961-1978. In: Anderson, O.D. (Ed.), Time Series Analysis, vol. 1. North-Holland, Amsterdam, pp. 203-226.

Taylor, S.J., 1994. Modelling stochastic volatility: a review and comparative study. Math. Finance 4, 183-204.

Taylor, S.J., 2005. Asset Price Dynamics, Volatility and Prediction. Princeton University Press, Princeton, NJ.

Yu, J., 2004. On leverage in a stochastic volatility model. J. Econometrics 127, 165-178.

Yu, J., Yang, Z., Zhang, X., 2006. A class of nonlinear stochastic volatility models and its implications for pricing currency options. Comput. Statist. Data Anal. 51, 2218-2231.

Zaffaroni, P., 2004. Stationarity and memory of $\operatorname{ARCH}(\infty)$ models. Econometric Theory 20, 147-160.

Zaffaroni, P., 2005. Whittle estimation of exponential volatility models. Manuscript. 